\title{
Ulrike $\mathrm{Haß}$
}

\section{Verfahren der Quellenverarbeitung in Zedlers Universal-Lexicon Aller Wissenschaften und Künste (1732-1754)}

\section{Fragestellung, Methode und Terminologie}

Ich befasse mich mit Enzyklopädien, weil sie eine lange und mächtige Tradition der Wissensvermittlung und -dokumentation darstellen (vgl. Stammen/ Weber 2004). Ich gehe davon aus, dass die Aufgabe der Wissensvermittlung mit spezifischen sprachlichen Strategien, Textroutinen (Feilke 2010) oder -mustern einhergeht, die weitgehend unabhängig vom fachlichen Gegenstand sind. Nach Feilke sind Textroutinen

\begin{abstract}
funktional bezogen auf rekurrente kommunikative Aufgaben; literal sind diese Routinen, soweit sie typisch oder - wie im Fall der Überschrift - sogar spezifisch für eine Kommunikation mittels schriftlicher Texte sind. Sie haben eine ausdruckseitig saliente Gestalt, die mehr oder weniger lexikalisiert sein kann. Ausdruckseitig können sie strukturell idiomatische Komponenten enthalten, können aber auch syntaktisch und semantisch völlig regulär sein. Die Salienz setzt gleichwohl eine ausdruckseitige Typisierung und Musterhaftigkeit voraus, die stets semiotisch pars pro toto auf ein Gebrauchschema [sic] mitverweist, das die Inhaltsseite der Prozedur ausmacht. Dadurch haben literale Prozeduren ein Kontextualisierungspotential. (Feilke 2010: 4)
\end{abstract}

Wie der bekannte Topos der ,Zwerge auf den Schultern von Riesen“ (Merton 1980) andeutet, ist eine der zentralen Eigenschaften wissenschaftlicher Texte die Lektüre und Verarbeitung vorhandener Wissensquellen zu einem neuen Wissenstext. Genau dies tun wissensvermittelnde Enzyklopädien, und zwar vermehrt und systematischer als andere wissenschaftliche Textsorten. Wo, wenn nicht in dieser Textsorte, könnte man sprachliche Routinen für Quellenbezüge erwarten? Die Textroutinen des Umgangs mit Quellentexten sind Thema dieses Beitrags. Dazu gehören aber nicht nur bestimmte Formulierungsmuster, sondern - vorgelagert die Frage nach den medialen Praxen: Welche Quelle wurden genutzt? Wurden sie genannt? Wurden sie kopiert oder umgeschrieben? Wurde ausgewählt, wurde kompiliert? Wurden Quellen diskutiert und miteinander verglichen?

Diesen Fragen soll hier am Beispiel von Zedlers Universallexikon nachgegangen werden, das von 1732 bis 1754 in 63 Bänden und 4 Supplementbänden

Ulrike Haß, Universität Duisburg-Essen, Institut für Germanistik, Berliner Platz 6-8, 45127 Essen, E-Mail: ulrike.hass@uni-due.de

○ Open Access. (๑) 2018 Ulrike Haß, publiziert von De Gruyter. (c) BY-NC-ND Dieses Werk ist lizenziert unter der Creative Commons Attribution-NonCommercial-NoDerivatives 4.0 Lizenz. 
erschien. Damit ist es der Frühaufklärung zuzurechnen. Es kann als Digitalisat der Bayerischen Staatsbibliothek durchsucht werden. ${ }^{1}$

Es gibt fast noch keine linguistischen Untersuchungen zu Zedlers Universallexikon, ${ }^{2}$ wohl aber verschiedene kulturwissenschaftliche, v. a. die Arbeit von Ulrich Johannes Schneider (2013), die auch einen Forschungsbericht zu Zedler enthält. Darauf habe ich mich für diesen Beitrag gestützt. Des Weiteren setze ich empirisch an und vergleiche zwei ausgewählte Quellenoriginale (s.u.) mit einigen Artikeln des Zedler, um die Frage nach den medialen Praxen der Quellenverarbeitung beantworten zu können. Anschließend habe ich einige typische Formulierungen der Bezugnahme auf diese und andere Quellen stichprobenartig über mehrere Bände analysiert, um Formulierungsmuster in ihren Funktionen festzustellen.

Doch zunächst gilt es, ein terminologisches Problem anzusprechen. Es gibt eine große Bandbreite geläufiger Bezeichnungen, um zu erfassen, was das enzyklopädische Schreiben bzw. was ein popularisierender Text ganz allgemein mit seinen Quellen macht. Die unten präsentierte willkürliche Sammlung enthält diese Bezeichnungen, von denen einige nicht nur im Exposee zu der hier dokumentierten Tagung (vgl. die Einleitung in diesem Band), sondern auch bei Schneider (2013) und in den Arbeiten der sog. Transferwissenschaft von Antos et al. ${ }^{3}$ vorkommen.

Redaktion
Übersetzung
Transformation
Transfer
Wissensproduktion
Wissenszirkulation
Systematisierung
Verarbeitung
Textimport
Neuordnung
speichern
abschreiben
transportieren
repetieren
umordnen

verständlich, zugänglich machen
interpretieren
selegieren
disponieren
formatieren
tradieren
extrahieren
ausschlachten
konstruieren
,fressen‘
ausschreiben
kompilieren
übernehmen
umschreiben
paraphrasieren

1 Es gibt zwei Suchoptionen: Einmal im Lemmabestand über alle Bände hinweg. Zum anderen eine Volltextsuche; letztere ist nur bandweise möglich.

http://www.zedler-lexikon.de/index.html?c=suchen\&suchmodus=volltext\&l=de (09. 10. 2018). 2 Nachfolgend spreche ich abkürzend von „Zedler“, wenn das gesamte Werk gemeint ist. 3 Vgl. zuletzt den Sammelband Antos \& Wichter (2005) in der Reihe Transferwissenschaften des Verlags Peter Lang. 
Einige dieser Bezeichnungen implizieren, Wissen ginge durch z. B. den wissenschaftspopularisierenden bzw. enzyklopädischen Prozess irgendwie unverändert hindurch und erhielte lediglich eine andere sprachliche oder mediale ,Hülle'. Auch unser Alltagskonzept von Information geht genau davon aus und entspricht damit der Container-Metapher des von der Nachrichtentechnik inspirierten Kommunikationsmodells von Shannon in den 1940er/1950er Jahren. ${ }^{4}$ Tatsächlich ist es m. W. aber noch nicht gelungen, diese unveränderte Kernsubstanz zu identifizieren.

Bei genauerer Betrachtung ist offensichtlich, dass hier kein Transfer oder Transport von etwas stattfindet, sondern vielmehr eine Art der Transformation, die etwas Neues schafft. ${ }^{5}$ Damit kommen von den o.g. Bezeichnungen am ehesten Wissensproduktion, Neuordnung oder Umordnung, wenn nicht gar (Neu-)Konstruktion in Betracht, wenn man den Prozess der Wissensvermittlung konzeptualisiert. Dies wird von Ludwig Jäger theoretisch eingehend reflektiert und mit dem Begriff der Transkriptivität gefasst, den ich deshalb auch für das wissenschaftliche bzw. enzyklopädische Schreiben vorschlagen möchte. Ein wesentliches Argument für die Nutzbarmachung des Transkriptivitätsbegriffs auch für eine Wissenschaftsgeschichtsforschung sind die Konsequenzen, die er für das Verständnis des ,kollektiven Gedächtnisses“ hat, ${ }^{6}$ ohne das eine historische Wissenschaftssprachforschung kaum denkbar ist.

Nach Jäger (2009) besteht der Prozess der Transkription gewissermaßen aus drei medialen Komponenten: Ausgangsbasis ist das „Präskript“, das im vorliegenden Falle des Zedler aus diversen (mono- oder multimodalen) Quel-

4 Kritisch dazu: Januschek (2005: 135-138).

5 Vgl. ausführlich hierzu Behrs, Gittel \& Klausnitzer (2013).

6 Jäger (2004: ohne Seite): „Beim gegenwärtigen Stand meiner Argumentation kann ich nun meine These, daß sich das kulturelle Gedächtnis nicht nur skriptural, sondern in einem weiteren Sinne über Verfahren der Intra- und Intermedialität konstituiert, so reformulieren: Kollektive Gedächtnisse werden nicht erst in den rezenten telematischen Wissensgesellschaften, sondern auch bereits in den skriptural kontaminierten Kulturen der Gutenberg-Galaxis und ihren präskripturalen Vorgängerkulturen (einschließlich der reichen Übergangsformen) immer in medialen Dispositiven adressiert, die verschiedene mediale Operatoren zueinander in Beziehung setzen. Der in den historischen Speicher kulturellen Wissens sedimentierte Sinn kann also nur in Verfahren semantisch aktiviert werden, d.h. affirmiert, über- und umgeschrieben oder auch arkanisiert und rearchiviert werden, die mindestens zwei Medien miteinander ins Spiel bringen: Diese treten dann entweder einseitig oder wechselseitig zueinander in die Beziehung der Auslegung, der Kommentierung, der Explikation und der Paraphrase etc., wobei sich die Sprache - gerade auch in ihrer mündlichen Diskursivität - als eine ständig verfügbare strategische Semantisierungsreserve erweist, ohne die die Verwandlung von implizitem (archiviertem) in expliziten (kommunikativ adressierten) Sinn, also die ,Lektüre in den Texturen des kulturellen Gedächtnisses, schwerlich gelänge.“ 
lentexten besteht. Am Ende steht das „Skript“, das heißt der mono- oder auch multimodale Text, den wir als Lexikonartikel (wieder-)erkennen. Das entscheidende und prozesshafte Mittelglied nennt Jäger „Transkript“; es ist in seinen symbolischen Mitteln wie Sprache oder Bild greifbar, zu denen auch (usuelle) Textroutinen oder (okkasionelle) sprachliche Verfahren bzw. Strategien zählen. Das Transkript ist an ein „mediales Dispositiv“ (Jäger 2004), d.h. an die Gesamtheit unterschiedlicher Rahmungen sprachlicher Kommunikationsakte gebunden (vgl. Jäger 2004: Anm. 21), hier an das Dispositiv der Enzyklopädie. Die Quellen werden in der Weise lesbar gemacht, dass sie zum enzyklopädischen Artikel werden. ${ }^{7}$ Der Prozess der Transkription wird in Enzyklopädien gelenkt durch die im weitesten Sinne medialen Bedingungen wie Textsortenerwartung, strukturelle Vorgaben wie das Alphabet, Quellenverfügbarkeit, praktische wie ökonomische Arbeitsbedingungen, Sprachkompetenz und die „transkriptive Intelligenz“ (Jäger 2009: 12) der Schreiber.

Ich gehe in meinem Beitrag nachfolgend auf die Suche nach regelhaften und über die Enzyklopädie hinaus wirkenden, möglicherweise bis heute weiter tradierten transkriptiven Verfahren enzyklopädischer Schreiber. M.a. W.: Anstatt nach einem schimärenhaften ,Inhalt‘ und seiner ,neuen Hülle‘ zu fragen, arbeite ich dasjenige heraus, was die Transkripte selbst über das enzyklopädische Schreiben verraten, und zwar fokussiert auf den Umgang mit Quellen. ${ }^{8}$

7 Wegen der komplexen Argumentation sei ein etwas längeres Zitat erlaubt. Jäger (2009: 11) fasst zusammen: „(1) Transkribieren lässt sich als ein Prozess der Konstitution von Skripten aus Präskripten beschreiben. Die Pointe dieses Prozesses besteht dabei darin, dass die Transkription in dem offenen Netzwerk von in einer gewissen Hinsicht unlesbaren Präskripten einen oder mehrere Ausschnitte fokussiert, ihnen eine semantische Ordnung gibt und sie so als Skript - in den Status der Lesbarkeit versetzt. (2) Weiterhin hat sich gezeigt, dass das Verhältnis zwischen Transkript und Skript nicht das einer Abbildung ist, weil die Transkription das Skript in gewissem Sinne erst generiert. Lesbarkeit war in der semantischen Hinsicht, die die Transkription eröffnet, zuvor keine Eigenschaft der durch das Transkribieren fokussierten Präskripte und insofern existierten diese auch noch nicht als (lesbare) Skripte. (3) Zugleich ist aber deutlich geworden, dass die konstitutive Abhängigkeit des Skriptes von seinem Transkript nicht als schlichte Derivation verstanden werden darf: Vielmehr wird das Skript insofern zu einer autonomen Bewertungsinstanz für die Angemessenheit der Transkription, als es zugleich den Raum für Postskripte öffnet, in denen die Angemessenheit der durch die Transkription behaupteten Lektüre in Frage gestellt werden kann.“

8 In Haß (2015) habe ich mich mit der Herausbildung typisch enzyklopädischer Textstrukturen und in Haß (2016) mit Verfahren der Begriffsreflexion in historischen Enzyklopädien befasst. 


\section{Transkriptive Verfahren, mediale Praxen und Textroutinen im Einzelnen}

Schneider (2013) hat Zedlers Quellen weitgehend, aber nicht erschöpfend ermittelt. Die Tabelle in Schneider (2013: 39) zeigt drei Quellenarten:

- über 20 (Fach-)Lexika, von denen mindestens zehn vollständig und ohne Offenlegung in das Universallexicon hineinkompiliert worden sind,

- partiell verarbeitete, teilweise lateinische Fachtexte v.a. des 17. Jahrhunderts, die vermutlich ihrerseits auch antike Quellen verarbeitet haben; unter diesen Fachtexten scheinen solche mit Überblicks-, Handbuch- oder Lexikoncharakter zu dominieren (Schneider 2013: 81),

- Chroniken und Zeitungen bzw. Zeitungsexzerpte, die geeignet sind, aktuellere Themen zu erschließen (Schneider 2013: 122).

Offensichtlich haben die Autoren des Zedler Quellen bevorzugt, die ihrerseits bereits aktiv Präskripte transkribiert haben; man muss also mit mehrstufigen Transkriptionen rechnen, ohne dass sie aufzuklären sind. Thematische Schwerpunkte in Zedlers Universallexicon liegen bei Biografie und Geografie (Schneider 2013: 153); Letzteres schließt Geschichte und Rechtsaspekte mit ein. In Artikeln $\mathrm{zu}$ diesen Themenbereichen werden erkennbar andere und überhaupt weniger Quellen verarbeitet, denn die Autoren scheinen sich damit besser auszukennen, während in naturwissenschaftsnahen Bereichen eher auf fachlexikalische Quellen zurückgegriffen wird.

Über Zedlers Autoren und ihre Arbeitsorganisation ist fast nichts bekannt. Die Artikel selbst zeigen keinerlei Systematik; nichts deutet auf redaktionelle Leitlinien oder dergleichen hin. Es gibt Indizien dafür, dass viele externe Autoren anonym zugeliefert und eigene Werke einer Zweitverwertung zugeführt haben. Autoren wurden z. T. öffentlich angeworben; dies hat offensichtlich v. a. bei Artikeln zu sächsischen Personen, Geschlechtern und Städten gut funktioniert, wie einzelne Textvergleiche gezeigt haben (Schneider 2013: 126, 148). Z. B. schrieb Chefredakteur Ludovici alle Artikel zu Christian Wolffs Philosophie und plagiierte dabei reichlich sich selbst, d.h. seine beiden Bücher zu Wolff (Schneider 2013: 175).

Immerhin wurde ab Band 19 im Jahr 1738 straffer und durch Ludovici als einzigem Redakteur kontrolliert gearbeitet. Bei vier Bänden oder 1000 Bögen pro Jahr hatte er 30 bis 40 Artikel täglich durchzusehen (Schneider 2013: 172). Hinweise auf drucktechnische Zwänge finden sich v. a. am Ende von Druckbögen und am Bandende. Der hohe Arbeitsdruck für Artikelautoren ist indirekt an Redundanzen im Artikel, aber auch an z. T. uneinheitlichen Benennungen 
im selben Artikel (Japaner neben Japaneser) erkennbar. Dieser hohe Arbeitsdruck macht es wahrscheinlich, dass sich arbeitsökonomische Routinen herausgebildet haben, aber auch, dass Willkür und Zufälligkeiten unrevidiert blieben.

Empirisch habe ich mich mit zweien der Quellen und ihren Spuren im Zedler näher befasst:

- erstens mit dem Allgemeinen Oeconomischen Lexicon von Zincke (17441764) und

- zweitens mit einer Sammlung thematisch ausgewählter und zwischen 1718 und 1730 quartalsweise gedruckt erschienener Zeitungsmeldungen, bei Zedler zitiert als Breßlauer Sammlungen oder Kanolds Sammlungen.

Letzteres wurde zum Vergleich gewählt, weil es sich nicht um eine lexikalische Quelle handelt. Bei der Nutzung lexikalischer Quellen liegt es ja nahe, die Lemmata zuzuordnen und lediglich den Artikeltext auf die eine oder andere Weise anzupassen. Schwieriger oder anders sieht es aus, wenn die Quelle nicht alphabetisch geordnete Teiltexte, sondern wie hier eine chronologisch kalendarische Ordnung von Wissenstexten enthält. Die Quelle stellt im Falle von Kanolds Sammlungen auch eine andere Textsorte dar, die im Titel als ,Geschichten“ und im Text selbst gelegentlich als „annales“ bezeichnet wird und die erkennbaren sprachlichen Merkmale zeitgenössischer Zeitungen aufweist (vgl. Haß-Zumkehr 1998). An diesem Beispiel wird unten deutlich werden, dass diese Art von Geschichten oder Berichten als ergänzende oder illustrative Einzelfälle behandelt werden, die der ergänzenden Lektüre empfohlen werden, nicht aber als Basis für Verallgemeinerungen dienen.

Nachfolgend werden sechs Arten transkriptiver Verfahren der Zedler-Autoren näher erläutert.

\subsection{Quellennennung}

Erwartungsgemäß werden Autoren und Quellen häufig genannt, z. T. abgekürzt und syntaktisch integriert. Syntaktische Integration ist so außerordentlich häufig, dass man dies vielleicht als das Standardverfahren bezeichnen kann, für das sich Textroutinen bilden konnten, z. B.

Und ist auch nicht glaublich, daß ein eintziges Thier sey, so im Feuer leben könnte; obwohl Plinius I.XI, c.36. solches von etlichen Thieren bewähret, die er Pyrales oder Pyraustas nennet. (Zedler Bd. 33, 1742, Sp. 651)

Insgesamt seltener als syntaktisch integrierte Quellenangaben, aber oft bei juristischen, theologischen und historischen Themen stellt der Lexikonartikel 
eine veritable Literaturdiskussion dar, z. B. zum „Rußischen Recht“ (Schneider 2013: 136). Die Literaturdiskussionen machen keinen Hehl aus den vielen Lücken im Wissen der Zeit, aus den Unsicherheiten und den abgestuften Wahrscheinlichkeiten. Hier wird ein differenziertes Arsenal epistemischer Ausdrucksmittel eingesetzt. Meist bezieht der Lexikonautor nicht Stellung, führt die Diskussion nicht $\mathrm{zu}$ einem argumentativen oder bewertenden Schluss (Schneider 2013: 141). Schon eher wird gelegentlich Unparteilichkeit demonstriert, indem ein Ereignis nach verschiedenen Quellen in zwei verschiedenen Versionen dargestellt wird.

Relativ selten finden sich Literaturangaben am Schluss eines Artikels, die Schneider (2013: 117) für Lektüreempfehlungen, nicht für Quellennachweise hält. Dagegen sprechen Beispiele wie das des langen Artikels „Tockenburg“ (Toggenburg), an dessen Ende ${ }^{9}$ eine umfangreiche und sehr genaue Quellenliste steht, die weit mehr Titel umfasst als die, die im Artikel direkt zitiert werden. Mir scheinen die hier verwendeten Formulierungen wie etwa

\footnotetext{
Wahrhafftiger u. gründlicher Entwurf, worauf das [...] nunmehro lange Zeit obgeschwebte Streit-Geschäfft eigentliche beruhe, 1710. in 4. Gründl. Information von denen Toggenburger Freyheiten und Gerechtigkeiten, $u$. den daher entstandenen Streitigkeiten mit dem Abt von St. Gallen, 1713, in Fol. u. in 4. Neue Europäische Fama XCII. Th. p. 701. u.f. (Zedler Bd. 44, 1745, Sp. 1076)
}

doch eher dem Nachweis zu dienen, dass der Autor gründliche Kenntnisse eingebracht hat, als zu weiterer Lektüre anzuregen.

\subsection{Literaturdiskussion}

Bei den Literaturdiskussionen wird oft von differenzierten epistemischen Mitteln Gebrauch gemacht, die nachfolgend aus einem Abschnitt des Artikels „Lucas, der Mahler“ (Zedler Bd. 18, 1738, Sp. 653-656) exzerpiert und zusammengestellt sind. In dem Textabschnitt geht es um ein Christus-Bild, das der Evangelist Lukas möglicherweise gemalt hat. Typische quellenkommentierende Konstruktionen sind:

- beruffen sich auf alte Aufschriften

- der vermeinte Haupt-Zeuge ist

- Darinne schreibet er

- daß von [Textname] ietzt nur zusammengelesene Stellen, welche X herausgegeben hat, vorhanden sind

9 Hier zur Grafschaft Toggenburg, Zedler Bd. 44, 1745, Sp. 1076. 
- daß diese aus X und andern Schriftverfassern gezogen

- der andere Zeuge ist Y, wie X in [Textname] behauptet

- dieser X schreibt

- Man hat aber hierbey zu erwegen, dass viele Schriften X untergeschoben oder verändert sind, daß er selbst ein nichtswürdiger Schriftsteller sey

- der dritte Zeuge ... dieser aber schreibt nichts mehr hiervon als: man sagt, Lucas habe das Bild gemahlet

- Der vierdte Zeuge ist X, welcher in [Textname] schreibt, man sage, daß Lucas gemahlet habe. Dieser Geschichtsschreiber weiß keinen andern Grund anzuführen, als die Leute sagen.

Man sieht, wie detailliert der Autor die Schichtung der Quellen wiedergibt und wie genau er dabei auf die Verlässlichkeit und Wahrscheinlichkeit der Aussage achtet. Das Verfahren zieht sich noch über etliche weitere Seiten.

Einerseits ist zu erkennen, dass der Autor damit Werturteile möglicherweise religiös-ideologischer Natur zu verbinden trachtet - er scheint manche Autoren nicht $\mathrm{zu}$ mögen. Andererseits kann man zusehen, wie er das eher Wahrscheinliche vom weniger Wahrscheinlichen einer überlieferten Aussage zu trennen versucht - eine Textbewegung, die ans Goldwaschen erinnert: Viel Quellenmaterial wird umgewälzt und am Ende bleibt ein Körnchen ,Wahrheit‘ übrig. Das Verfahren ähnelt sehr dem in zeitgenössischen Zeitungen derselben Zeit. ${ }^{10}$ Den Lesern sollen aber keineswegs nur die aus Quellen selegierten Wahrheits-Körner vorgelegt werden - vielmehr ist der Vorgang von Diskussion und Selektion unerlässliches Element des enzyklopädischen Artikels.

\subsection{Zitat, Zitatkommentierung und Zitatmarkierung versus Referat}

Zitate werden in Zedler anders als heute üblich zu Beginn jeder Zeile mit einem doppelten Anführungszeichen unten gekennzeichnet. Man sieht also sehr gut, welchen Druckraum ein Zitat im Umgebungstext einnimmt, auch wenn es mitten in der Zeile beginnt und endet. Es wird in Zedler viel, v. a. ,graue Literatur zitiert, die kaum gedruckt und in Bibliotheken greifbar gewesen sein wird, z. B. das Gutachten eines Musikers über einen anderen:

Der Chur-Sächsische Capellmeister, Heinrich Schütz, hat ein Gutachten darüber verfertiget, und ihm diesen Titel beigelegt [...]. In der Vorrede meldet der Verfasser folgendes: „[...]““ (Zedler Bd. 18, 1738, Sp. 215, zu „Löw, Johann Jacob“)

10 Mit zahlreichen Formulierungsbeispielen: Haß-Zumkehr (1998: 97-102). 
An anderen Orten werden über mehrere Seiten hinweg Friedensverträge, Denkschriften und andere rechtlich-historische Texte zitiert, z. B.:

Tóckóly ließ auch zu dem Ende eine sehr nachdenckliche Schrift verfertigen, und solche unter der Hand durch einen seiner Bedienten, in diesem Königreich ausstreuen. Wo nicht schon alle Vortheile auf Seiten des Kaysers gewesen wären, sollte sie ausser Zweifel eine grosse Würckung gethan haben. Der Inhalt davon war kürtzlich dieser: „[...]“ (Zedler Bd. 44, 1745, Sp. 1056)

Erstaunlich daran ist, dass solche Quellen durchweg als ereignisbeeinflussende Faktoren historisch-politischer Zusammenhänge behandelt werden, indem die Art und Weise ihrer Verbreitung und ihrer Rezeption zitateinleitend mitgeteilt werden, z. B. „dass die Deputirten den Tractat durchaus nicht annehmen wollten“ (Zedler Bd. 44, 1745, Sp. 1056-1057). Den Quellen, die die ,Welt verändern‘, gehört die besondere Aufmerksamkeit der Schreiber. Der komplette, über 16 Spalten reichende Abdruck eines Friedensvertrags von 1710, also nicht lang zurückliegend, wird eingeleitet durch Schilderung der vorangehenden Verhandlungen und derjenigen Situation, die Auslöser des Textes war (ebd.).

Fachliteratur hingegen wird eher referiert als zitiert. Redekennzeichnendes Mittel ist hier oft der Konjunktiv II, nicht der Konjunktiv I, z. B.:

Gerhard Blasius, der weltberühmte holländische Professor, schreibet in seiner Anatomia Animalium, so zu Amsterdam in 4to. gedrucket worden, von einem kleinen Afrikanischen Löwen, so nur neun monath alt gewesen, folgendes: Es wäre nemlich der Unter-Leib von dem Wasser aufgelauffen gewesen. [...] Die Därme wären [...] aufgeschwollen gewesen. [...] Das Netz hätte der Magen an sich gezogen gehabt“ usw. (Zedler Bd. 18, 1738, Sp. 219)

Den Autor einer Quelle zu rühmen, wie hier, dient sicherlich der Legitimierung des Inhalts. Die häufige Mitteilung äußerer Texteigenschaften wie des Formats (,in Quarto‘) und des Druckorts sollen womöglich belegen, dass der Lexikonautor die Quelle selbst in Händen hatte oder sich zumindest tatsächlich mit dem Werk befasst hat. Vielleicht ist dies ex negativo ein Indiz für die häufige Nutzung indirekter, aus zweiter und dritter Hand schöpfender Quellen. Die eigenhändige direkte Rezeption einer Quelle wäre dann der abweichende Fall, auf den mit Nennung materialer Texteigenschaften wie des Formats dezent hingewiesen wird.

\subsection{Textkopie}

Der Vergleich mit Zinckes Oeconomischem Lexicon zeigt, was auch die ZedlerLiteratur sagt, dass viel plagiiert wurde. Da die Autoren des Universallexicons 
anonym waren, sei der Anreiz selbstständig zu formulieren gering gewesen, so Schneider (2013: 41). Exakte, unveränderte Kopien kompletter Artikel sind immer verdeckt, nicht gekennzeichnet. Hier Auszüge aus dem Artikel „Bau“:

\begin{tabular}{lll}
\hline & Zincke s.v. „Bau“ (Spalte 231) & Zedler s. v. „Bau“ (Bd. 3, 1733, Sp. 704) \\
\hline a & heissen bei der Jägerey die Löcher & nennen die Jäger das Loch \\
b & Dachse, Füchse, Caninichen, Fisch-Ottern & der Fuchs und Dachs \\
c & von dem Fuchse ist bekannt & ist zwar die Gewohnheit des Fuchses \\
d & sich darein logiren kann & sich drein logiret \\
e $\quad$ Da aber der Fuchs & Wenn er aber \\
f $\quad$ muss er nothwendig seinen Bau & und sich also unumgänglich genötiget \\
& & siehet, zu seiner Sicherheit eine Wohnung \\
g $\quad$ Man findet auch öffters & zu haben \\
h $\quad$ Dergleichen machen nur junge Füchse & Auch findet man offtermals \\
\hline
\end{tabular}

Dass man aber mühevoll versuchte, die Textkopie zu verschleiern, indem kleine und ganz unbedeutende Textänderungen vorgenommen wurden, ${ }^{11}$ könnte an der hohen Leipziger Buchproduktivität gerade auch auf dem Feld von Lexika gelegen haben. Aus der Vorrede zu Zedler Bd. 19 (1739) erhellt, dass konkurrierende Leipziger Lexikon-Verleger um Verschlechterung ihrer Marktchancen fürchteten und deshalb versuchten, juristisch gegen Zedler vorzugehen (Schneider 2013: 159).

Das Beispiel des polysemen Lemmas „Bau“ zeigt aber, dass wohl nur einzelne Artikel, höchst selten ganze Lemmastrecken kopiert wurden, obwohl auch für Letzteres ein Beispiel gegeben werden kann: Zincke setzt zwei separate Lemmata „Faß“ an, einmal $F a \beta$ als Bier-/Weingefäß und einmal $F a \beta$ als Maßeinheit. Zedler übernimmt diese Ansätze, fügt aber ein drittes Lemma Faß für Gefäß im allgemeinen Sinne und in übertragenem Gebrauch mit Anführung von Bibelstellen hinzu; dieser Artikel ist sehr kurz (Zedler Bd. 9, 1734, Sp. 292).

Die textlichen Veränderungen bei der Kopie von „Bau“ lassen keinerlei Systematik erkennen, außer in (b) einen Ansatz zur Ersetzung von Vielfalt durch generische Singularformen und bei (c) und (d) die Tendenz, epistemisch markierte Formulierungen in Tatsachenbehauptungen zu verwandeln. Die Änderungen machen den Text autoritativer, lexikonartiger. Diese Tendenz hat eine Parallele in der Verarbeitung von Reisebeschreibungen für Länderartikel

$11 \mathrm{Zu}$ ganz ähnlichen Ergebnissen führt auch der Vergleich der Artikel zum Lemma „Faß“ bei Zincke und Zedler; auf Demonstration wird hier verzichtet. 
im Zedler, die Schneider (2013) untersucht hat. Hier haben Änderungen der Vorlage darin bestanden, aus Einzelbeobachtungen und einzelnen Erlebnissen im Zedler generische Aussagen zu machen. Hat der Reisebericht ein paar spanische Frauen schüchtern erlebt, dann schreibt das Lexikon, spanische Frauen seien schüchtern (Schneider 2013: 121).

Ganz anders gelagert ist der Fall, dass Fremdwörter der Quelle im Zedler gezielt verdeutscht wurden, wie dies für die ansonsten verdeckt exakte Kopie aus Hanns Friedrich von Flem(m)ings „Vollkommenen Teutschen Soldaten“ (1726) festgestellt wurde (Schneider 2013: 151). Dies ist möglicherweise motiviert durch die Vorrede zu Band 1 des Zedler, die auch das Wissen außerhalb der „hohen Schulen“, das von „Künstler[n] und Handwercker[n]“, „Hauswirthe[n] und Kaufleute[n]“ gebraucht werde, zu repräsentieren ankündigt (zit. n. Schneider 2013: 74).

Man muss also von einer Vielfalt von Gründen für das plagiierende Kopieren von Quellentexten ausgehen. Die folgenreichsten Gründe scheinen die der ökonomischen Konkurrenz zu anderen Nachschlagewerken und des Zeitdrucks gewesen zu sein. Leichte Veränderungen sind erkennbar der Anschärfung des Textsortenprofils als Informationsautorität zuzuschreiben. Zugleich aber gibt es die Bemühung, breitere Adressatenkreise außerhalb der gelehrten Welt anzusprechen, zumindest nicht auszuschließen.

\subsection{Auswahl und Abwahl}

Keineswegs immer wurden Artikel aus Zincke übernommen, auch wenn es nahegelegen hätte. Die Lexikon-Quelle Zincke mit ihrem ökonomischen Schwerpunkt genügte den Autoren des Zedler bei manch einem eher juristisch oder historisch relevanten Lemma offensichtlich nicht. In Abwahl und Neuformulierung wird die Perspektive auf den Gegenstand des Lemmas gewechselt, wie das Beispiel des Rechenfehlers zeigt. Der „error calculi“ wird im ökonomischen Lexicon Zinckes (Bd. I, Sp. 671) auf neuneinhalb Zeilen aus der Perspektive von Verwaltern und Verwaltungspraxis dargestellt. In Zedler hingegen wird er als „Exceptio Erroris Calculi“ in den juristischen Fachdiskurs der vielen „Exceptiones“ eingeordnet und $\mathrm{zu}$ anderen Rechtsbegriffen in Beziehung gesetzt; der Artikel ist hier 36 Zeilen lang (Zedler Bd. 8, 1734, Sp. 2281). Während Zincke im Rechtsinstrument des Error Calculi eine Erleichterung und Absicherungsmöglichkeit rechnungsführender Verwalter sieht, scheint der Verwalter bei Zedler auf der Anklagebank zu sitzen, denn das Rechtsinstrument wird äußerst argwöhnisch geschildert. Kurze Auszüge sollen diesen deutlichen Perspektivenwechsel veranschaulichen: 
Zincke Bd. I, Sp. 671

Error calculi, heisset ein Irrthum, oder ohne Vorsatz begangener Fehler, in denen Zahlen einer Rechnung. Salvo errore calculi, pflegen Rechnungs=führende Beamte, Verwaltere und dergleichen, zu Ende ihrer Rechnungen zu setzen, und durch diese Cautel zu verhüten, daß [...] solches ihnen nicht nachtheilig fallen und zum Schaden gereichen möge.
Zedler Bd. 8, 1734, Sp. 2281

Exceptio Erroris Calculi, eine Ausrede wegen beschehener Mißrechnung, oder daß ein Irrthum in der Rechnung vorgegangen sei [...]. Es wird solche zu denen zerstörlichen Ausflüchten zwar gezählet, [...] weil sie von einem Contract dependiret, und kann solche Ausflucht, ob ihr schon renunciret worden, dennoch obponiret werden [...] es kann aber diese Exception nicht gebrauchet werden, wenn 1) $[\ldots]$ 2) $[\ldots . .$, 3) $[\ldots]$ 4) $[\ldots]$

Die hier ausgelassenen Textstellen enthalten zahlreiche Hinweise auf juristische Quellen mit Angabe von Paragraphen. Der Autor des Zedler argumentiert als Jurist und verweist dabei auch auf teils unterschiedliche Rechtsauffassungen. Der Text ist sichtbar von juristischen Fachwörtern durchzogen, die - was hier nicht dargestellt werden kann - wie üblich in Antiqua gesetzt sind. Pointiert formuliert: Die Sicht des Prozessgegners, der das Instrument des Error Calculi abzuwehren hat, verdrängt hier die Sicht des Verwalters, der zu ihm Zuflucht nehmen möchte.

Hintergrund und Motiv solcher Abwahl von Quellenelementen scheint über die individuelle Expertise des Autors hinaus eine besondere und gewollte inhaltliche Ausrichtung von Zedlers Universallexikon zu sein. Schneider (2013: 77-79) hat per Zuordnung jedes Artikels zu einem von sechzehn Wissensbereichen ermittelt, dass der quantitative Schwerpunkt auf Personen, v. a. Adeligen und Gelehrten, Geografie, Recht, Politik, Geschichte lag. Die Ökonomie (im damals üblichen weiteren Begriffsumfang) hingegen, die durch Zinckes Lexicon repräsentiert wird, rangiert quantitativ, d. h. nach Anzahl der Artikel, etwa im Mittelfeld der Wissensbereiche. Selbst Medizin und der Bereich Kunst, Literatur, Bildung sind mit mehr Artikeln vertreten als die Ökonomie. Quantitativ hinter dem Bereich Ökonomie rangieren Handwerk, Botanik, Zoologie, Mathematik und einige deutlich kleinere Bereiche.

Mit der gleichen inhaltlichen Tendenz werden - durch Kommentierung und Einbringen individuellen Wissens - auch Texterweiterungen vorgenommen. Dies scheint besonders bei Artikeln zu sächsischen Städten und Personen der Fall zu sein (Schneider 2013: 125-127 und 140). Die eigene Stadt wird ausführlicher und unter Einbeziehung individueller Erfahrungen beschrieben. Persönliche Nähe und individuelles Autorwissen führen zu längeren Artikeln. 


\subsection{Kombination: Matrixtext und Einschübe}

Das letzte der hier zu behandelnden transkriptiven Verfahren stellt gewissermaßen die Kombination aller vorherigen Verfahren dar und scheint die effizienteste und damit vermutlich häufigste Strategie zu sein. Zugleich zeigt diese spezielle Form der Kombination vielleicht auch Tendenzen der Konventionalisierung der unter 2.1 bis 2.5 beschriebenen Strategien.

Das kombinierende Verfahren kopiert und/oder zitiert Quellen ausschnittweise, kommentiert und kombiniert sie in einer neuen Weise. Dabei spielt stets eine einzige Quelle eine besondere Rolle, die ich hier als Matrixquelle bezeichne. ${ }^{12}$ Der Matrixtext macht den größten Teil des Artikels aus und liefert ihm i. d.R. auch den Anfang. Andere, kürzere Lexikon-Artikel eignen sich hierfür grundsätzlich besser als andere Quellengattungen wie z. B. Zeitungstexte. In den Matrixtext werden dann Textauszüge anderer Quellen eingeschoben. Dabei werden die Textteile unterschiedlicher Herkunft typografisch und syntaktisch so gut integriert, dass das ,Puzzle، nicht auffällt. Die Quellen der eingeschobenen Textteile sind entweder fachlich vertiefende, gelehrte Texte oder sie bereichern den Matrixtext um praktische und um Anwendungsaspekte, wie es im Falle von essbaren und/oder heilenden Pflanzen naheliegt. Das von Schneider beschriebene Beispiel des Artikels „Vanille“ stimmt in der Struktur der Artikel zu anderen Pflanzen nicht nur im Zedler, sondern auch in historisch späteren Enzyklopädien auffallend überein. ${ }^{13}$

Es liegt auf der Hand, dass Zeitungsquellen oder Reisebeschreibungen eher für unterhaltsame oder Neugier weckende Einschübe geeignet erschienen. Während sich Fachlexika relativ gut als Matrixtext zu eignen scheinen, ist eine Quelle wie Kanolds Sammlung, die ihrerseits eine offensichtliche Ausbeutung und Neunutzung von Zeitungstexten darstellt, eher für Texteinschübe geeignet. Zeitungen und Chroniken liefern singuläre Vorkommnisse, Beobachtungen und Beispiele; ein Matrixtext hingegen muss generische Aussagen enthalten oder leicht in generische Aussagen zu überführen sein. Dies sei abschließend an einem Beispiel der Quellenverwertung veranschaulicht, bei dem der Autor des Zedler sowohl auf Zinckes Lexikon als auch auf Kanolds Sammlungen zurückgegriffen hat. Der Artikel in Zincke umfasst wenige Zeilen, die nachfolgend komplett wiedergegeben sind:

Mehl=Thau, ist eine von der Nacht=Kälte und denen Morgen=Frösten zusammengebackene gifftige Feuchtigkeit, welche bey kalten Nächten auf Gras, Kräuter und Laub fället,

12 Grafisch dargestellt am Beispiel des Eintrags „Vanille“ bei Schneider (2013: 156).

13 Vgl. dazu Haß (2015) zur Artikelstruktur zum Lemma „Linse“ in Zedler, Brockhaus und Meyer's des späten 19. Jahrhunderts bis hin zur Wikipedia. 
und von der darüber aufgehenden Sonne gerinnet oder anbäcket, oder gar wie in eine weisse Spinnenwebe verkehret wird; davon das Laub einläufft, und viel schädliches Ungeziefers an Würmen und Mücken aushecket, das Gras aber, welches mit solchem scharfen Zeuge beschmitzet ist, dem Vieh übel gedeihet. Den Mehl=Thau wollen etliche vertreiben, wenn sie einen Rauch von Mist, Horn und feuchtem Stroh machen, daß ihn der Wind durch den Garten treibe. Was zarte Gewächse sind, mag so wohl den Mehl=Thau als den Reiff mit subtiler Ansprengung eines frischen Wassers abspülen, dass sie beyde nicht so gefährlich eingreiffen können; es muss aber solches vor der Sonnen Aufgang geschehen, denn so sie einmahl von der Sonne beschienen sind, ists hernach vergeblich. (Zincke Bd. 2, 1764, Sp. 1858)

Mit dieser Lexikon-Quelle beginnt auch der Autor des Zedler seinen Artikel (Bd. 20, 1739, Sp. 269-275); eine auffallende Änderung stellt der Einschub dar, der nachfolgend unterstrichen ist. Er markiert damit den Artikel, zumindest bis zur Nennung der ersten Spezialquelle, als eine Art kollektives oder KonsensWissen, weil, so kann man interpretieren, die von ihm benutzte Quelle ein Lexikon ist und durch diese kollektiv-konsentische Art des Wissens charakterisiert ist:

Mehl=Thau, ist, nach dem gemeinsten Vorgeben derer Natur=Kündiger, eine von der Nacht=Kälte und denen Morgen=Frösten zusammen gebackte gifftige Feuchtigkeit, welche bey kalten Nächten auf Gras, Kräuter und Laub fällt, und von der darüber aufgehenden Sonne gerinnet oder anbäckt, oder gar wie eine weisse Spinne=Webe verkehrte wird; wovon das Laub einläufft, und viel schädliches Ungeziefer an Würmern und Mücken aushecket, das Gras aber, welches mit solchem scharffen Zeuge beschmitzet ist, dem Vieh übel gedeyet. Den Mehl=Thau wollen einige vertreiben, wenn sie einen Rauch von Mist, Horn und feuchten Stroh machen, daß ihn der Wind durch den Garten treibe. Was zarte Gewächse sind, mag man sowohl den Mehl=Thau mit subtiler Ansprengung frischen Wassers abkühlen, daß beyde nicht so gefährlich angreiffen können; doch muß es vor der Sonnen Aufgang geschehen: Denn woferne sie einmal von der Sonne beschienen worden, ist es hernach vergeblich.

An dieser Stelle endet der Text der Quelle (Zincke). Zedlers Autor fährt aber ohne Absatz oder sonstige Markierung weiter fort wie folgt, indem er weitere, offensichtlich noch nicht konsensfähige Ansichten über das Phänomen aneinanderreiht und dabei die Uneinigkeit auch kritisiert; einige der referierten Ansichten werden Autoritäten zugeordnet, andere nicht:

Einige sagen auch, es wären die Blätter mit einer Art Mehl befallen; andere meynen, es sey ein süsser Honig=Thau, und werde von dem lateinischen Worte Mel also genennet. So pflegt man auch insgemein zu sagen, es falle ein Mehl=Thau, da es nur bei hellem Sonnen=Schein um den Mittag ein wenig Staub regnet, welches sodenn alles lebendig würde und die Bäume verdürbe. Nun kommen wohl die meisten welche den Mehl=Thau nennen darüber überein, dass es ein schädlicher Thau sey, davon nicht nur die Gewächse und ihre Früchte verderben, sondern auch das Vieh ansteckende Seuchen bekommet. 
Man pflegt auch von demselben die Verschrumpffung und Verderbung der Blätter, da sie als mit Rost beflecket und in Fäulniß und Verdorrung gebracht, herzuleiten. Ein berühmter Schweitzer, Nahmens Scheuchzer sagt in der Natur=Wissenschaft II. Theil, c. 24, n. 14, vom Mehl=Thau ausdrücklich, er falle nicht eigentlich vom Himmel herunter, sondern sey ein durch die Sonnenhitze und vorhergehende Regen aufgelöseter, ausgekochter und durch die äußersten Röhrlein gewisser Pflantzen flüssender Nahrungs=Saft, der sich daher mehr an den untern als obern Theilen der Blätter finde, und nach Verschiedenheit der Gewächse eine purgirende oder andere Würckungen habe. Wie aber solches zugehe, davon schweigen die Gescheutesten stille oder überlassen es andern zu untersuchen. Einige aber erklären es folgendergestalt, es würden durch den Mehl=Thau, als eine klebriche Materie, die Löcher der Gewächse verstopffet, dass die Ausdünstungen nicht ordentlich geschehen könnten, davon sie denn nothwendig beschädiget werden müsten. Bes. Boernero Physic. p. 247. [...] (Zedler Bd. 20, 1739, Sp. 269-275).

Daran anschließend folgen drei Spalten Text mit zahlreichen weiteren, teilweise als Vermutungen gekennzeichneten Aussagen zum Thema mit jeweils einzelnen Quellenangaben. Dann wird explizit aus Kanolds Sammlungen zitiert:

Die Sammlung Breßlauischer Medicorum von Natur= und Medicin=Geschichten, 1722, Monath September. Class. IV. Art. V. P. 279. u. ff. bringen dieses davon bey: Unter denen Physicalischen Begebenheiten, so, bey der heut zu Tage vermeinten Vollkommenheit der Physic, dennoch vielen Widersprüchen unterworffen, ist nicht die geringste, die Äthiologie der jährlich häufig zu observirenden Mehl= und Honig=Thaue, unwidersprechlich zu erklären. Ich will, um Weitläufftigkeit zu vermeiden, mich nicht gäntzlich in dieses latifundium einlassen, sondern nur meine unmaßgebliche Gedancken entwerffen, was ich von dem Mehl=Thau bis daher observiret, vielleicht möchte durch fleissige Collationierung anderer Observationum die wahre Aethiologie endlich zu finden seyn. Die Historie der Sache bestehet darinnen, daß im Jahr 1722. allhier in den den meisten Gärten an denen Bäumen, vornemlich an denen Birnen, die Blätter und Früchte erst gelbe und fleckicht, dann schrumpficht, mit allerhand Tuberculis seu Excrescentiis, ut sic dicam, callosis, besetzt, befunden worden. [...] Dies wären also meine flüchtige Gedancken, so ich die über dieses Jahr vom Mehl=Thau betroffne Bäume hege, die, ob ich sie zwar für unumstößlich nicht halte, so glaube doch, wer folgende Zeit den Verstand der Sachen genauer und fleißige untersuchet [...] und dadurch vielleicht mit der Zeit ein Mittel erlangen, in gewissen Fällen dergleichen Verderbnissen vorzubeugen, oder abzuhelffen.

Dies ist das Ende des Zitats aus Kanold, das im Original drei Seiten umfasst (Seite 297-300) und interessanterweise in der ersten Person des Beobachters und Zeugen geschrieben ist. Der Text des Artikels in Zedler geht aber, ohne das Zitatende zu markieren, also auch, ohne den Wechsel von der ersten in die dritte Person zu kennzeichnen, noch etwa 45 Zeilen weiter, bevor auch Zedlers Autor seinen Artikel beendet. Die ,Überleitung' lautet ähnlich wie diejenige, mit der von Zinckes Lexikonartikel aus weiterformuliert wurde, ${ }^{14}$ wie folgt: „Andere Gedancken sind dahin gegangen, daß [...]“.

14 Siehe oben: „Einige sagen auch ..., andere meynen“. 
Damit kann im Rahmen des in diesem Fall methodisch Möglichen bestätigt werden, dass Matrixtextverfahren, d. h. das Verfahren der kombinierenden Kompilation verschiedener Quellen in einem Artikel mit der Heraushebung einer der Quellen als Matrix, neben Neuschreiben und exakten Kopien als das häufigste Verfahren der Quellen-Transkription in Enzyklopädien gelten. ${ }^{15}$ Der Matrixtext muss nicht lang sein; seine Funktion besteht darin, kollektives oder konsentisches Wissen in generischer Weise zu präsentieren. Die hinzugefügten Quellen haben jeweils die Funktion der beobachtenden oder argumentierenden Einzelstimme. Zedlers Lexikon-Autor versucht mitunter kritisch, den Chor dieser Stimmen zu referieren. Der Matrixtext hingegen scheint immer ein Text $\mathrm{zu}$ sein, der dieser kritischen Infragestellung entzogen ist.

\section{Textroutinen und Syntagmen}

Die verschiedenen Weisen, mit denen Quellentexte im Zedler verarbeitet wurden, waren meistens an sprachlichen Mitteln erkennbar, denn Zedlers Autoren beziehen sich oft explizit auf das, was sie gelesen haben. Daher soll es nachfolgend um die Frage gehen, ob es usuelle Textroutinen oder syntagmatische Bausteine gibt, die die Funktion der Redewiedergabe in Zedlers Enzyklopädie erfüllen und die möglicherweise das enzyklopädische Schreiben der nachfolgenden Jahrhunderte geprägt haben.

Ausgangspunkt sei Zedlers Artikel „Sprache, Rede, loquela“, mit dem sich Wichter (1996) v.a. inhaltlich befasst hat. Folgende Auszüge aus dem achtzehn Spalten umfassenden Artikel zeigen Formulierungen der Quellenreferenz und der Quellenkommentierung:

[...] welches in Sonderheit Amman in seiner Dissertation: de loquela umständlich ausgeführet hat [...] wovon Amman in der angeführten Dissertation [...] insbesondere Exempel gegeben hat. Wolfens vernünfftige Gedancken von dem Gebrauche der Theile in dem Menschen etc. p. 495 u.f. [...] Dieses beweiset unter andern de la Motte im zweyten Theile seiner Chirurgie, p. 176 mit folgendem Exempel: [...] so schreibet doch Riolan in seiner Anthropographia [...] De Jußieu hat in den Mémoire de l'académie de science eine Anmerkung von [...] beygebracht [...] Ein Exempel, dass [...] führet Friedel an, in seinem XVI medicinischen Bedencken [...] (Zedler Bd. 39, 1744, Sp. 402, 403).

Wie in den oben in Abschnitt 2 diskutierten Beispielen ist auch hier zu erkennen, in welch hoher Dichte diverse lexikalische Mittel der Redewiedergabe ver-

15 Schneider (2013: 152) referiert eine unveröffentlichte Staatsexamensarbeit von Duesberg (1978), die für den Bereich der Poetik anhand von 126 Artikeln im Zedler herausfand, dass sie mehrheitlich nach dem von mir Matrixtextverfahren genannten kompiliert worden waren. 
wendet wurden. Weitere, z. T. epistemisch markierte Beispiele aus zufällig gewählten anderen Artikeln sind:

- $\quad$ in der Vorrede meldet der Verfasser folgendes

- X schreibet in seiner Y ... folgendes

- der Inhalt davon war kürzlich dieser

- Von ihrem Anfang bringet X verschiedene Meynungen hervor, unter welchen diese der Wahrheit am nächsten zukommen scheinet, daß sie nehmlich ...

Von diesem induktiv gewonnenen lexikalischen Material der Quellenreferenz und -kommentierung gehe ich aus und untersuche systematischer deren Usualität, indem ich die Häufigkeit auffälliger Lexeme stichprobenartig in neun der 63 plus vier Supplementbände des Zedler ermittelt habe. Untersucht wurden folgende Lexemformen:

- meldet / melden, schreib(e)t / schreiben, bring(e)t / bringen, Exempel, angemerk(e)t, Anmerkung, Inhalt(s), ausführt / ausführen / ausgeführet.

Die Volltextsuche wurde in folgenden Bänden durchgeführt: 1, 3, 8, 18, 19, 30, 40, 60, Supplementband 4. Bei der Bestimmung der Bände kam es mir auf eine breite und gleichmäßige Streuung über die Entstehungszeit an. Aussagen zur Usualität eines Lexems oder eines Syntagmas können sich nur auf absolute Trefferzahlen stützen. Wegen der unkorrigierten und daher unzuverlässigen Texterkennung des Zedler-Digitalisats kann man nicht ernsthaft quantifizieren, sondern lediglich Tendenzen zu finden versuchen.

Das häufigste Verbum dicendi im Zusammenhang mit Quellennennung ist schreibet/schreiben, und zwar mit namentlich genannten Einzelautoren als Subjekt. Usuell ist das Syntagma:

- (wie) X schreibt

meldet/melden ist im Zedler eher selten, hingegen besonders häufig in Zeitungen des 18. Jahrhunderts (vgl. Haß-Zumkehr 1998: 85 u. 117), was auf eine textsorten-funktionale Differenzierung hindeutet.

anmerken wird oft zur Textdeixis oder mit indefiniten Subjekten (man, einige, andere) gebraucht (und eher selten), um bestimmte Meinungspositionen der Quellen (Präskripte) im Lexikon (Skript) abzuschwächen:

- man hat/einige, andere haben angemerk(e)t.

Das Verbalsubstantiv Anmerkung ist formelhaft gebunden an Untertitel von Quellenschriften, fällt also aus den Textroutinen der Quellenverarbeitung heraus:

- $\quad$ mit (gelehrten, ausführlichen) Anmerkungen herausgegeben/übersetzt von X. 
ausführen/ausgeführt wird noch eher für nicht-sprachliches Tun gebraucht und ist nicht typisch für die hier interessierenden Funktionen der Quellenverarbeitung.

Inhalt kommt fast immer als Genitiv-Konstruktion vor, wenn eine Quelle nicht wörtlich zitiert, sondern ihr Gehalt zusammengefasst werden soll:

- Schreiben/Brief des/dieses/folgendes Inhalts: ...

Die attributlose Konstruktion des Inhalts scheint für Rechtstexte typisch zu sein und ,wandert' so auch in den Zedler.

Exempel ist nicht selten, hat aber keine redekommentierende Funktion, sondern referiert auf einen Fall oder ein Ereignis, der bzw. das zu argumentativen Zwecken angeführt wird. Typisch ist:

- zum Exempel.

Es wurde ja deutlich, dass manche Quellen (Reisebeschreibungen und Zeitungen) an die deskriptiv-explikative Themenentfaltung der Enzyklopädie angepasst wurden; mit dem Ausdruck Exempel können solche Quellen in den Matrixtext eingebracht und zugleich einer mehr oder weniger impliziten generischen Aussage zugeordnet werden.

\section{Fazit}

Zedlers Autoren haben vielfältige und differenzierte Verfahren der Quellenverarbeitung eingesetzt, die von verdecktem Plagiat bis hin zur expliziten Literaturdiskussion reichen. Diese Verfahren des Umgangs mit Quellentexten sind ihrerseits aus Quellen übernommen, nicht neu erfunden worden. Die für Enzyklopädien typischen Verfahren mischen die Lexikon-Autoren aber neu und verändern sie so, dass sie der Textfunktion des Lexikons gerecht werden und den Zusammenhang zwischen Textfunktion und sprachlichen Realisierungsformen textmusterspezifisch ausprägen. Zugleich wird eine Ausdifferenzierung der sprachlichen Mittel der Quellenreferenz zwischen Lexikontext und anderen Textgattungen wie Zeitungen und Reisebeschreibungen angebahnt.

Geeignete bzw. beliebte Quellen des Zedler gehören zur gelehrten, z.T. fachspezifischen Literatur, aber vor allem zu den älteren Lexika. Es konnte gezeigt werden, dass und warum Lexika eher verdeckt plagiiert und Texte der Spezialliteratur eher explizit diskutiert werden. Aus exemplarischen Beschreibungen und Beobachtungen, wie sie Zeitungen und Reisebeschreibungen zur Verfügung stellen, werden im Zedler generische Aussagen, v. a. wenn der Quellentyp des Lexikons greifbar war. 
Der enorme Publikationsdruck der Autoren des Zedler hat dazu geführt, dass transkriptive Verfahren routinisiert wurden und dass in deren Gefolge bestimmte sprachliche Konstruktionen und Formulierungsroutinen entstanden, die nicht nur für lexikontypisch gehalten werden konnten, sondern auch in anderen wissenschaftlichen Texten der Folgezeit wiederzufinden sein könnten.

\section{Literatur und Quellen}

Antos, Gerd \& Sigurd Wichter (Hrsg.) (2005): Transferwissenschaft. Wissenstransfer durch Sprache als gesellschaftliches Problem (Transferwissenschaften, Bd. 3). Frankfurt a. M.: Peter Lang.

Behrs, Jan, Benjamin Gittel \& Ralf Klausnitzer (2013): Wissenstransfer. Konditionen, Praktiken, Verlaufsformen der Weitergabe von Erkenntnis (Berliner Beiträge zur Wissens- und Wissenschaftsgeschichte, Bd. 14). Frankfurt a. M.: Peter Lang.

Feilke, Helmuth (2010): „Aller guten Dinge sind drei!“ Überlegungen zu Textroutinen und literalen Prozeduren. In: Iris Bons, Thomas Gloning \& Dennis Kaltwasser (Hrsg.), Fest-Platte für Gerd Fritz. Gießen 17. 05. 2010. http://www.festschrift-gerd-fritz.de/files/ feilke_2010_literale-prozeduren-und-textroutinen.pdf (09.10. 2018).

Haß-Zumkehr, Ulrike (1998): „Wie glaubwürdige Nachrichten versichert haben“. Formulierungstraditionen in Zeitungsnachrichten des 17. bis 20. Jahrhunderts (Studien zur deutschen Sprache, Bd. 13). Tübingen: Narr.

Haß, Ulrike (2015): Kochen als enzyklopädische Herausforderung. In: Hermann Cölfen (Hrsg.), Brot und Spiele - Sprache, Kochen und Essen (Osnabrücker Beiträge zur Sprachtheorie, Heft 87). Duisburg: Redaktion: OBST, 17-44.

Haß, Ulrike (2016): Wissenschaftssprache in der Vermittlung von Wissen durch Enzyklopädien. In: Eglè Kontutytė \& Vaiva Žeimantienè (Hrsg.), Sprache in der Wissenschaft. Germanistische Einblicke (Duisburger Arbeiten zur Sprach- und Kulturwissenschaft, Bd. 111). Frankfurt a. M. u. a.: Peter Lang, 13-29.

Jäger, Ludwig (2004): Transkription - zu einem medialen Verfahren an den Schnittstellen des kulturellen Gedächtnisses. In: Trans. Internet-Zeitschrift für Kulturwissenschaften 15. http://www.inst.at/trans/15Nr/06_2/jaeger15.htm (09.10. 2018).

Jäger, Ludwig (2009): Transkriptivität. Zur medialen Logik der kulturellen Semantik. In: transkriptionen. Newsletter des Kulturwissenschaftlichen Forschungskollegs Medien und kulturelle Kommunikation 10, 8-12. http://kups.ub.uni-koeln.de/2711/ (09. 10. 2018).

Januschek, Franz (2005): Aufstieg und Fall von Kommunikationsmodellen. Kommunikation gibt's das? In: paradigms lost (Osnabrücker Beiträge zur Sprachtheorie, Heft 69). Duisburg: Redaktion: OBST, 131-152.

Kanold, Johann (1724): Sammlung von Natur- und Medicin-, wie auch hierzu gehörigen Kunst- und Literatur-Geschichten. Erfurt 1718-1730. Daraus: Der neun u. zwantzigste Versuch Sommer-Quartal, 1724. http://reader.digitale-sammlungen.de/resolve/display/ bsb10499080.html (09.10. 2018). Der dreyßigste Versuch Herbst-Quartal, 1724. http:// reader.digitale-sammlungen.de/resolve/display/bsb10499081.html (09. 10. 2018).

Merton, Robert King (1980): Auf den Schultern von Riesen: ein Leitfaden durch das Labyrinth der Gelehrsamkeit. Frankfurt a. M.: Syndikat. 
Schneider, Ulrich Johannes (2013): Die Erfindung des allgemeinen Wissens. Enzyklopädisches Schreiben im Zeitalter der Aufklärung. Berlin: Akademie Verlag.

Stammen, Theo \& Wolfgang E. Weber (Hrsg.) (2004): Wissenssicherung, Wissensordnung und Wissensverarbeitung. Das europäische Modell der Enzyklopädien. Berlin: Akademie-Verlag.

Wichter, Sigurd (1996): „Sprache, Rede, Loquela“ in Zedlers Universal-Lexicon. In: Hans Höfinghoff u. a. (Hrsg.), Alles was recht war. Rechtsliteratur und literarisches Recht. Festschrift für Ruth Schmidt-Wiegand zum 70. Geburtstag. Essen: Item, 235-246.

Zedler, Johann Heinrich (1732-1754): Großes vollständiges Universal-Lexicon Aller Wissenschaften und Künste. 64 Bände und 4 Supplementbände. Halle, Leipzig: Zedler. http://www.zedler-lexikon.de/index.html?c=startseite\&l=de (09.10. 2018).

Zincke, Georg Heinrich (1744-1764): Allgemeines Oeconomisches Lexicon Darinnen nicht allein Die KunstWörter und Erklärungen dererjenigen Sachen, welche theils in der Oeconomie überhaupt, theils insonderheit in einer vollständigen Landwirtschafft und Haushaltung von Acker, Feld, Holtz, Hopffen, Obst, Wein und GartenBau, Wiesewachs, Fischerey, Jägerey, Bierbrau. Leipzig 1744. Band 2: 1764. http://www.mdz-nbnresolving.de/urn/resolver.pl?urn=urn:nbn:de:bvb:12-bsb10299856-6 (09. 10. 2018). 